\title{
Phytohormone responses in pepper (Capsicum annuum L.) leaves under a high density of aphid infestation
}

\author{
Victoria Florencio-Ortiz ${ }^{1, *}$, Ondřej Novák², José L. Casas ${ }^{1}$ \\ ${ }^{1}$ Unidad Asociada IPAB (UA-CSIC), Instituto Universitario de Investigación CIBIO (Centro \\ Iberoamericano de la Biodiversidad), University of Alicante, Carretera de San Vicente del \\ Raspeig, s/n, E-03690 San Vicente del Raspeig, Alicante, Spain \\ ${ }^{2}$ Laboratory of Growth Regulators, Centre of the Region Haná for Biotechnological and \\ Agricultural Research, Institute of Experimental Botany AS CR \& Palacký University, \\ Šlechtitelů 11, CZ-78371 Olomouc, Czech Republic \\ * Corresponding author \\ E-mail: victoria.florencio@ua.es
}

\begin{abstract}
The time course response of selected phytohormones has been evaluated in sweet pepper plants (Capsicum annuum L.) submitted to a high density (200 aphids/plant) of aphid (Myzus persicae Sulzer) infestation. Abscisic acid (ABA), salicylic acid (SA), indole-3-acetic acid (IAA), and jasmonates (JAs), including jasmonic acid (JA), jasmonoyl-L-isoleucine (JA-Ile), and cisOPDA have been simultaneously identified and quantitated by UHPLC-MS/MS in pepper leaf tissue harvested at 3, 8 hours post-infestation (hpi), 1, 2, 4 and 7 days post-infestation (dpi). Infested plants showed a reduction in stem length at $7 \mathrm{dpi}$ and in the number of leaves and leaf width from 4 dpi onwards. JA and JA-Ile significantly increased very early (from 3 hpi) while SA only accumulated at $7 \mathrm{dpi}$. Despite the high density of infestation, the aphid-induced accumulation of JAs was much lower than the burst typically induced by chewing herbivores. On the other side, ABA peaked in aphid-infested plants at 2 and 4 dpi, while IAA content did not change significantly at any time point. Growth inhibition may be partially explained by the high levels of JAs found in aphid-infested plants. The possibility that the obtained results support the hypothesis of the aphid manipulation of plant metabolism is discussed.
\end{abstract}

\section{Keywords}

Aphid-plant interaction; UHPLC-MS/MS; Biotic stress Plant defence; Phytohormones

\section{Abbreviations}

This article has been accepted for publication and undergone full peer review but has not been through the copyediting, typesetting, pagination and proofreading process which may lead to differences between this version and the Version of Record. Please cite this article as doi: $10.1111 /$ ppl.13188 
ABA, abscisic acid; cisOPDA, cis-12-oxo.phytodienoic acid; IAA, indole-3-acetic acid; JA, jasmonic acid; JA-Ile, jasmonoyl-L-isoleucine; SA, salicylic acid

\section{Introduction}

As sessile organisms plants have no opportunity other than to adjust their growth and physiology in ways that optimize their reproductive success in a dynamic environment, where they are constantly attacked by a vast number of insects and pathogens and also submitted to several types of abiotic stresses (including high salinity, drought, or extreme temperature) (Dar et al. 2015, Berens et al. 2017, Howe et al. 2018). In nature, selective pressures shaped the evolutionary emergence of signalling networks that continuously integrate environmental signals into complex transcriptional responses that promote plant resilience (Howe et al. 2018). Phytohormones are key elements within these signalling networks. Changes in environmental clues induce changes in the quantity and composition of phytohormones to facilitate appropriate plant responses (Huot et al. 2014, Dar et al. 2015, Shigenaga and Argueso 2016, Berens et al. 2017).

Salicylic acid (SA) and jasmonates (JAs) including jasmonic acid (JA) and its derivatives, are traditionally recognized among the major plant defence hormones (Verma et al. 2016). SA is generally involved in the activation of defence response against biotrophic and hemi-biotrophic pathogens, whereas JA signalling is primarily involved in defence against insect herbivores and necrotrophic pathogens. SA and JA pathways act mostly antagonistically to each other, albeit their interaction is dependent both on concentration and genetic background, and together comprise the backbone of the hormonal regulation of plant immunity (Smith et al. 2009). However, in the past decade a substantial body of evidence has demonstrated that auxins, abscisic acid (ABA), ethylene, gibberellins (GAs), cytokinins (CKs), and brassinosteroids (BRs), typically associated with abiotic stress or developmental processes, are also key components of the immune response of plants. All these phytohormones tend to act interdependently through antagonistic or synergistic interactions (reviewed in Dar et al. 2015, Shigenaga and Argueso 2016, Verma et al. 2016, Berens et al. 2017, Karasov et al. 2017). Moreover, an emerging consensus highlights the close regulatory control of growth and defence by the plant immune signalling networks. Plant fitness is optimized when growth and defence are appropriately prioritized in response to both environmental and developmental cues. The JA 
pathway plays a key role in mediating growth-defence tradeoffs through crosstalk with other hormone pathways (Campos et al. 2014, Havko et al. 2016, Karasov et al. 2017, Züst and Agrawal 2017, Guo et al. 2018, Howe et al. 2018,).

Aphids are considered one of the most damaging pests in world agriculture. This is partially due to their capacity to reproduce parthenogenically along with a short generation time that allow them to reach high population densities. Moreover, aphids are able to colonize virtually any part of the plant, consume profuse amounts of photoassimilates from the phloem, alter source-sink relationships, vector more than 200 viral diseases and resist to a large number of insecticides (Giordanengo et al. 2010, Nalam et al. 2018). It is largely known that insect herbivory induces a rapid accumulation of the "wound hormone" JA (Koo and Howe 2009, Lortzing and Steppuhn 2016), phenomenon known as JA burst. This accumulation is of a large magnitude in the case of chewing insects (Tscharntke et al. 2001, von Dahl and Baldwin 2004, Diezel et al. 2009, Stork et al. 2009) but seems to be limited in the case of aphid infestation (Gosset et al. 2009, Mai et al. 2014, Stewart et al. 2016). It is in accordance with the slight mechanical damage that aphid feeding inflicts compared to chewing insects, as aphids access phloem sieve elements penetrating plant tissue with their stylet primarily via an apoplastic route (Giordanengo et al. 2010, Morkunas et al. 2011).

In a previous study we investigated ABA, IAA, SA and JAs accumulation in pepper (Capsicum annuum L.) leaves in response to a low density (20 aphids/plant) of green peach aphid (Myzus persicae Sulzer) infestation (Florencio-Ortiz et al. 2018b). In that case, aphid movement was restricted to individual leaves taking the advantage of clip cages. This approach allowed us to distinguish between local and systemic responses but, in turn, it does not allow achieve high densities of aphid infestation in the plant. Major responses were found in JAs whereas SA, ABA and the auxin indole-3-acetic acid (IAA) showed only some punctual responses. Moreover, these responses were moderate and manifested mostly at local level (Florencio-Ortiz et al. 2018b). Given that JA led to the production of defences and the inhibition of growth in a dosedependent manner (Baldwin et al. 1997, Schmelz et al. 2003, Havko et al. 2016) the prevention of the JA burst may have strong implications in plant defence responses to aphids. In this context, the present study was aimed to determine JAs, SA, ABA and IAA content in pepper plants under a high density (200 aphids/plant) of M. persicae infestation. This aphid density was observed tocause visible effects on plant growth. 


\section{Materials and methods}

\subsection{Plant material and aphid infestation}

C. annuum var. California Wonder seeds (Ramiro Arnedo S.A, Murcia, Spain) were germinated in plastic pots with a 1:1 mixture of peat (Prohumin potting soil, Projar S.A., Valencia, Spain) and vermiculite. Plants were watered three times a week and maintained in a growth chamber under a $16: 8 \mathrm{~h}$ photoperiod (day/night), $24^{\circ} \mathrm{C}$, and $70 \%$ relative humidity. A green peach aphid colony was maintained on pepper plants as described previously (Florencio-Ortiz et al., 2018a). Five weeks after sowing, pepper plants were sequentially infested with 200 wingless adult aphids to harvest leaves at 3, 8 hours post-infestation (hpi), 1, 2, 4 and 7 days post-infestation (dpi). Plants were infested sequentially (with the longer times of infestation initiated first)in order all plant tissue for phytohormone analysis culd be harvested at the same time, thus minimizing variability. Ten plants at each sampling time were randomly harvested and separated in five biological replicates. The same number of uninfested plants was used as a control. At the end of the experiment, insects were brushed off and the leaves were kept in liquid nitrogen during collection and thenstored at $-80^{\circ} \mathrm{C}$ for at least $48 \mathrm{~h}$ until freeze-drying. Tissue wasfinally grounded and stored at $4^{\circ} \mathrm{C}$ until extraction.

\subsection{Phytohormone extraction and UHPLC-MS/MS analysis}

Sample preparation and analysis was done according to Floková et al. (2014). Briefly, 3 mg (dry weight, DW) were used for each sample. Each biological replicate was extracted and analysed twice, giving two technical replicates, and the results of both were averaged. Six phytohormones including IAA, ABA, SA, and jasmonates (OPDA, JA and JA-Ile) were analyzed. The phytohormones were extracted using an aqueous solution of methanol $(10 \%$ $\mathrm{MeOH} / \mathrm{H} 2 \mathrm{O}, \mathrm{v} / \mathrm{v})$. A cocktail of stable isotope-labelled standards consisting of: $5 \mathrm{pmol}$ of $\left[{ }^{13} \mathrm{C}_{6}\right]$ IAA, 10 pmol of $\left[{ }^{2} \mathrm{H}_{6}\right] \mathrm{JA},\left[{ }^{2} \mathrm{H}_{2}\right] \mathrm{JA}-\mathrm{Ile}$, and $\left[{ }^{2} \mathrm{H}_{6}\right] \mathrm{ABA}, 20 \mathrm{pmol}$ of $\left[{ }^{2} \mathrm{H}_{4}\right] \mathrm{SA}$ and $\left[{ }^{2} \mathrm{H}_{5}\right]$ OPDA (all from Olchemim Ltd, Czech Republic) was added to each sample. The extracts were purified using Oasis HLB columns (30 mg/1 mL, Waters) and the analytes eluted with $80 \% \mathrm{MeOH} / \mathrm{H}_{2} \mathrm{O}$. After gently evaporation of $\mathrm{MeOH}$ under nitrogen stream, JA and its derivatives, $\mathrm{ABA}, \mathrm{SA}$ and IAA were analysed by ultra-high performance liquid chromatography-electrospray tandem mass spectrometry (UHPLC-MS/MS)using an Acquity 
UPLC $^{\circledR}$ System (Waters, Milford, MA, USA) equipped with an Acquity UPLC BEH C18 column (100 x $2.1 \mathrm{~mm}, 1.7 \mathrm{~m}$; Waters,Ireland), and the effluent was introduced into the electrospray ion source of a triple quadrupole mass spectrometer Xevo TQ-S MS (Waters, Milford, MA, USA). The analysed compounds and appropriate stable isotope-labelled internal standards were quantified in multiple ion monitoring mode (MRM) using the optimized MS conditions described in Floková et al. (2014).

\subsection{Plant growth measurements}

In order to follow the temporal evolution of plant growth under aphid infestation three different parameters were measured: stem length, number of expanded leaves and leaf width. At the beginning of the experiment ( 5 weeks after sowing), all plants presented two pair of leaves expanded. Thus, the parameter "leaf width" was measured on the emerging leaf of the third pair of leaves. Leaves were counted as expanded when their width was up to $1 \mathrm{~cm}$. Plants were measured at four different time points during the experiment (Figure 1). Twenty-five plants were included in each treatment of aphid infestation (2, 4 and 7 dpi) and the same number of plants was measured as a control.

\section{Statistical analysis}

The effect of aphid infestation on phytohormones content was assessed with Analysis of Variance (ANOVA) followed by a post hoc analysis to detect significant differences between times of aphid infestation. Assessment of the normality of data was conducted with ShapiroWilk test. Post-hoc tests were conducted with Tukey's HSD or Games-Howell, depending on whether or not Levene's test showed homogeneous variance. The effect of aphid infestation on stem length, leaf width and number of expanded leaves was assessed with Kruskal-Wallis test. Data analysis was conducted with the software IBM SPSS Statistics version 22. In all cases differences were considered to be significant at $P<0.05$.

\section{Results and discussion}

Herbivory induces the production of plant defensive compounds that exert direct toxic, antinutritional, or repellent effects on insects and also emit volatile organic compounds (VOCs) that work indirectly by attracting the insect's natural enemies (Howe and Jander 2008, Wang and 
Wu 2013, Campos et al. 2014, Havko et al. 2016, ). JA has become recognized as key regulator of inducible defences, including virtually all major classes of secondary metabolites (phenylpropanoids, polyketides, terpenoids, alkaloids and amino acid derivatives) as well as some defence proteins and volatile compounds (Wang and Wu 2013, Campos et al. 2014, Dar et al. 2015, Guo et al. 2018, Howe et al. 2018,). In the present study JA and its metabolite JA-Ile, the active form of JA, increased significantly from 3 hpi onwards in pepper plants in response to a high density (200 aphids/plant) of aphid infestation (Figure 2A and 2B). Consequently, the content of cis-OPDA, the JA precursor, showed a significant reduction at $1 \mathrm{dpi}$, recovering the control levels thereafter (Figure 2C). In the case of JA, the increase ranged from 5.5 to 14.7-fold and in the case of JA-Ile from 9.8 to 14.9-fold. Even though the accumulation in both JAs was higher than in a previous study using an initial lower density of aphids (Florencio-Ortiz et al. 2018b) it was still considerably lower than the JA burst commonly induced by chewing insects (Tscharntke et al. 2001, von Dahl and Baldwin 2004, Diezel et al. 2009, Stork et al. 2009). Some studies have demonstrated that the production of defences, including nicotine (Baldwin et al. 1997) and volatile compounds (Schmelz et al. 2003) occur in a JA dose-dependent manner. Thus, the fact that even a considerably high levels of aphid infestation did not trigger a strong JA accumulation in the plant, compared to chewing insects, may partially contribute to the success of aphids as crop pests. Interestingly, in the highly specialized phytophagy shown by gall-forming aphids the JA signalling pathway, and consequently the production of VOCs, seems to be compromised in gall tissue compared to the leaf tissue (Takei et al. 2015).

During feeding aphids secrete salivary proteins into the plant aimed to divert or suppress plant defences (Furch et al. 2015, Naessens et al. 2015, Mugford et al. 2016, Van Bel and Will 2016). Aphids have been suggested to induce the SA pathway as a decoy strategy for rerouting plant response towards an inefficient plant defence (Giordanengo et al. 2010, Morkunas et al. 2011). In the present study, SA levels in pepper plants remained unaltered as a consequence of aphid infestation until 7 dpi, when SA content in infested plants was 3-fold higher than in control plants (Figure 2D). Previous studies have also described a delayed after aphid infestation (Mohase and Van der Westhuizen 2002, Mai et al. 2014, Stewart et al. 2016, Florencio-Ortiz et al. 2018). Considering that SA has been proved to inhibit catalase and stimulate peroxidase activity (Mohase and Van der Westhuizen, 2002), thus providing a line of plant defence through the accumulation of reactive oxygen species (Sun et al. 2020), then the question arises as to whether this late SA induction may be part of the aphid strategy of plant- 
defence manipulation. The prevention of SA accumulation may be beneficial for aphids as it may hinder the hydrogen peroxide-mediated response of SA. The induction of the ABA pathway has been also suggested to be part of the aphid's decoy strategy to interfere with effective defences, given that $M$. persicae population growth is compromised on ABA-deficient Arabidopsis mutants (Hillwig et al. 2016). However, a recent study has determined that ABA contributes to soybean tolerance to the soybean aphid (Chapman et al. 2018). This highlights the importance of investigating specific plant-aphid systems and do not rely on the information based on model plants. In pepper plants ABA content tended to increase at late times of aphid infestation and was significantly higher than in control plants at 2 and 4 dpi (Figure 2D). Conversely, endogenous levels of IAA were not significantly changed in pepper plants in response to aphids coinciding with previous results (Florencio-Ortiz et al. 2018b) and despite the high aphid density employed in this study. The suppression of auxin signalling is considered to enhance tolerance to biotic and abiotic stresses whereas the activation of auxin signalling is often associated with susceptibility to pathogens (reviewed in Shigenaga and Argueso 2016 and Berens et al. 2017). Recent evidences suggest that, similarly to pathogens, auxin signalling disruption is associated with enhanced aphid resistance (Smith et al. 2010; Marimuthu and Smith 2012, Sattar et al. 2016).

It is well documented that herbivory and JA-triggered immunity are antagonistically linked to a potent inhibition of growth (Redman et al. 2001, Poveda et al. 2003, Heinrich et al. 2013, Attaran et al. 2014, Ferrieri et al. 2015, Machado et al. 2017, ). This phenomenon has been traditionally explained as the direct result of allocation costs, given the assumption that plants possess a limited pool of resources that can be invested either in growth or in defence resulting in a growth-defence trade-off. However, a substantial body of recent evidences highlight that growth restriction is an adaptive plant response resulting from regulatory crosstalk and may optimize the temporal and spatial expression of defence (Campos et al. 2014, Havko et al. 2016, Karasov et al. 2017, Guo et al. 2018, , Howe et al. 2018, Züst and Agrawal 2017). Plant growth rate is a relevant part of plant performance, at any point in time, and is a crucial measure to study resource allocation decisions in real time (Züst and Agrawal 2017). Stem length presents a good correlation with plant dry mass and has been used as non-destructive parameter to monitor the plant's investment into growth in Nicotiana attenuata (Heinrich et al. 2013, Machado et al. 2017). In the present study, a high density of aphid infestation caused a reduction in the stem length in pepper plants after 7 dpi compared to control plants (Figure 3). 
Also, both the number of leaves (Figure 4) and leaf width (Figure 5) were reduced compared to control plants and these effects were manifested from 5 dpi onwards. Although additional explanations (decreased photosynthesis or divergence of nutrients to aphids) cannot be ruled out, growth inhibition in aphid-infested pepper plants may be partially explained by the high levels of JAs induced in response to infestation. JAs have been shown to reduce leaf expansion (Attaran et al. 2014), stem elongation (Heinrich et al. 2013), biomass accumulation (Leone et al. 2014, Robson et al. 2010, Zhang and Turner 2008) and root elongation (Staswick et al. 1992, Feys et al. 1994). Interestingly, the ability of exogenous JA to inhibit root elongation occurs in a dose-dependent manner and is widely used as a simple quantitative bioassay of JA sensitivity (Havko et al. 2016). In the present study, plants belonging to 2 and 4 dpi treatments did not show any difference in growth (stem length, number of leaves or leaf width) compared to control plants (Figure 3). In an analogous experiment pepper plants were infested with an initial low aphid density of 20 aphids/ plant and neither difference in growth were observed at 2, 4, or 7 dpi compared to control plants (data not shown). It has been suggested that growth-defence tradeoffs occur in a context-dependent manner and may be manifested only when the flux of primary metabolites into defence pathways exceeds a certain likely high threshold (Guo et al. 2018), such as it seems to occur in our experimental system.

JA-induced plant growth inhibition takes place by different mechanisms, including the suppression of mitosis, the arrest of the cell cycle in G1 prior to the S transition, and the delay of the switch from the mitotic cell cycle to the endoreduplication cycle (Zhang and Turner 2008, Noir et al. 2013). But in addition to JAs, other hormones may be also implicated in the growth inhibition observed in the present study. ABA regulates numerous developmental processes and adaptive stress responses in plants (Cutler et al. 2010). Its role in plant growth is determined by dose and tissue sensitivity and ranges from stimulatory to inhibitory effects (Humplík et al. 2017). Interestingly, ABA signalling has been recently shown to repress Target of Rapamycin (TOR) kinase during times of stress contributing to the balance between plant growth and stress responses (Wang et al. 2018). Suppression of growth by SA is best illustrated by constitutive defence mutants, which typically have a dwarf plant phenotype (Huot et al. 2014). Moreover, one of the primary ways SA has been shown to inhibit growth is by suppression of auxin signalling (Huot et al. 2014) which was not altered in pepper plants under aphid infestation. However, in the present study SA accumulated in pepper plants only at 7 dpi (figure 2) while certain aspects of the growth reduction (number of leaves and leaf width) were visible 2 days 
earlier (figures 4 and 5, respectively). Thus, it would be interesting to examine the responses in other growth hormones that have been implicated in growth-defence tradeoffs, including CKs (Naseem et al. 2015, Albrecht and Argueso 2017) and BRs (Lozano-Durán et al. 2013, De Bruyne et al. 2014). But particularly important has been the wave of recent studies showing a crucial role for the JA-GA signalling crosstalk in regulating the growth-defence trade-off through direct physical interaction between JAZ and DELLA repressor proteins (Yang et al. 2012, Heinrich et al. 2013, Leone et al. 2014, Machado et al. 2017).

Research on model species has revealed that interactions between defence and growth, as well as abiotic and biotic stress, are often mediated by hormone crosstalk. However, different plant lineages appear to have specialized networking of phytohormone signalling pathways. Thus, basic knowledge gained from model species needs to be translated to crops (Berens et al. 2017). Plant productivity, yield and resistance to environmental stresses are currently the main points of interest for agriculture and plant biotechnology. Given their key role in plant fitness, metabolic engineering of phytohormones is receiving increasing attention for achieving these goals (Berens et al. 2017, Ciura and Kruk 2018). Our data provide new insights into relationships between plant growth and defence in crops.

\section{Authors contributions}

Victoria Florencio-Ortiz: Conceptualization, Investigation, Data curation, Formal analysis, Writing original draft, - Writing - review and editing.

Ondrej Novak: Methodology, Formal analysis, Writing - original draft, Writing - review and editing, Supervision.

José L. Casas: Conceptualization, Formal analysis, Writing - original draft, Writing - review and editing, Supervision, Project Administration.

\section{Acknowledgements}

This work was supported by the Ministerio de Economía y Competitividad of Spain, Project CGL2016-79054-R, by the University of Alicante grant UAFPU2013-5793 to VFO, and by the Ministry of Education, Youth and Sports of the Czech Republic - NPU I program with project LO1204. The present work is part of VFO PhD Thesis.

\section{Data availability statement}


Data sharing is not applicable to this article as all new created data is already contained within this article.

\section{References}

Albrecht T, Argueso CT (2017) Should I fight or should I grow now? The role of cytokinins in plant growth and immunity and in the growth-defence trade-off. Ann Bot 119: 725-735

Attaran E, Major IT, Cruz JA, Rosa BA, Koo AJK, Chen J, Kramer DM, He SY, Howe GA (2014) Temporal dynamics of growth and photosynthesis suppression in response to jasmonate signalling. Plant Physiol 165: 1302-1314

Baldwin IT, Zhang Z-P, Diab N, Ohnmeiss TE, McCloud ES, Lynds GY, Schmelz EA (1997) Quantification, correlations and manipulations of wound-induced changes in jasmonic acid and nicotine in Nicotiana sylvestris. Planta 201: 397-404

Berens ML, Berry HM, Mine A, Argueso CT, Tsuda K (2017) Evolution of hormone signaling networks in plant defense. Annu Rev Phytopathol 55: 401-25

Campos ML, Kang J-H, Howe GA (2014) Jasmonate-triggered plant immunity. J Chem Ecol 40: 657-675

Chapman KM, Marchi-Werle L, Hunt TE, Heng-Moss TM, Louis J (2018) Abscisic and jasmonic acids contribute to soybean tolerance to the soybean aphid (Aphis glycines Matsumura). Sci Rep 8: 15148

Ciura J, Kruk J (2018) Phytohormones as targets for improving plant productivity and stress tolerance. J Plant Physiol 229: 32-40

Cutler SR, Rodriguez PL, Finkelstein RR, Abrams SR (2010). Abscisic acid: emergence of a core signaling network. Annu Rev Plant Biol 61: 651-79

Dar TA, Uddin M, Khan MMA, Hakeem KR, Jaleel H (2015). Jasmonates counter plant stress: A review. Environ Exp Bot 115: 49-57

De Bruyne L, Höfte M, De Vleesschauwer D (2014). Connecting growth and defense: the emerging roles of brassinosteroids and gibberellins in plant innate immunity. Mol Plant 7: 943-959

Ferrieri AP, Arce CCM, Machado RAR, Meza-Canales ID, Lima E, Baldwin IT, Erb M (2015) A Nicotiana attenuata cell wall invertase inhibitor (NaCWII) reduces growth and 
increases secondary metabolite biosynthesis in herbivore-attacked plants. New Phytol 208: 519-530

Feys BJF, Benedetti CE, Penfold CN, Turner JG (1994) Arabidopsis mutants selected for resistance to the phytotoxin coronatine are male sterile, Insensitive to methyl jasmonate, and resistant to a bacterial pathogen. Plant Cell 6: 751-759

Floková K, Tarkowská D, Miersch O, Strnad M, Wasternack C, Novák O (2014) UHPLCMS/MS based target profiling of stress-induced phytohormones. Phytochemistry 105, 147-157.

Florencio-Ortiz V, Sellés-Marchart S, Zubcoff-Vallejo J, Jander G, Casas JL (2018a) Changes in the free amino acid composition of Capsicum annuum (pepper) leaves in response to Myzus persicae (green peach aphid) infestation. A comparison with water stress. PLOS ONE 13(6): e0198093. https://doi.org/10.1371/journal.pone.0198093.

Florencio-Ortiz V, Novák O, Casas JL (2018b) Local and systemic hormonal responses in pepper (Capsicum annuum L.) leaves under green peach aphid (Myzus persicae Sulzer) infestation. J Plant Physiol 231: 356-363

Furch ACU, van Bel AJE, Will T (2015) Aphid salivary proteases are capable of degrading sieve-tube proteins. J Exp Bot 66: 533-539

Giordanengo P, Brunissen L, Rusterucci C, Vincent C, van Bel A, Dinant S, Girousse C, Faucher M, Bonnemain J-L (2010) Compatible plant aphid interactions: how aphids manipulate plant responses. C R Biol 333: 516-523

Guo Q, Major IT, Howe GA (2018) Resolution of growth-defense conflict: mechanistic insights from jasmonate signalling. Curr Opin Plant Biol 44: 72-81

Havko NE, Major IT, Jewell JB, Attaran E, Browse J, Howe GA (2016) Control of carbon assimilation and partitioning by jasmonate: an accounting of growth-defense tradeoffs. Plants 5:7 https://doi:10.3390/plants5010007

Heinrich M, Hettenhausen C, Lange T, Wünsche H, Fang J, Baldwin IT, Wu J (2013) High levels of jasmonic acid antagonize the biosynthesis of gibberellins and inhibit the growth of Nicotiana attenuata stems. Plant J 73: 591-606

Hillwig MS, Chiozza M, Casteel CL, Lau ST, Hohenstein J, Hernández E, Jander G, Macintosh GC (2016) Abscisic acid deficiency increases defence responses against Myzus persicae in Arabidopsis. Mol Plant Pathol 17: 225-235. 
Howe GA, Jander G (2008) Plant immunity to insect herbivores. Annu Rev Plant Biol 59: 4166

Howe GA, Major IT, Koo AJ (2018) Modularity in jasmonate signaling for multistress resilience. Annu Rev Plant Biol 69: 387-415

Humplík JF, Bergougnoux V, Van Volkenburgh E (2017) To stimulate or inhibit? that is the question for the function of abscisic acid. Trends Plant Sci 22: 830-841 https://dx.doi.org/10.1016/j.tplants.2017.07.009

Huot B, Yao J, Montgomery BL, He SY (2014) Growth-defense tradeoffs in plants: a balancing act to optimize fitness. Mol Plant 7: 1267-1287

Karasov TL, Chae E, Herman JJ, Bergelson J (2017) Mechanisms to mitigate the trade-off between growth and defense. Plant Cell 29: 666-680

Koo AJK, Howe GA (2009) The wound hormone jasmonate. Phytochemistry 70: 1571-1580

Leone M, Keller MM, Cerrudo I, Ballar CL (2014) To grow or defend? Low red: far-red ratios reduce jasmonate sensitivity in Arabidopsis seedlings by promoting DELLA degradation and increasing JAZ10 stability. New Phytol 204: 355-367

Lortzing T, Steppuhn A (2016) Jasmonate signalling in plants shapes plant-insect interaction ecology. Curr Opin Insect Sci 14: 32-39

Lozano-Durán R, Macho AP, Boutrot F, Segonzac C, Somssich IE, Zipfel C (2013) The transcriptional regulator BZR mediates trade-off between plant innate immunity and growth. eLife 2:e00983. https://doi.org/10.7554/eLife.00983

Machado RAR, Baldwin IT, Erb M (2017) Herbivory-induced jasmonates constrain plant sugar accumulation and growth by antagonizing gibberellin signalling and not by promoting secondary metabolite production. New Phytol 215: 803-812

Marimuthu M, Smith CM (2012) Barley tolerance of Russian wheat aphid (Hemiptera:Aphididae) biotype 2 herbivory involves expression of defense response and developmental genes. Plant Signal Behav 7: 382-391

Mohase L, Van der Westhuizen AJ (2002) Salicylic acid is involved in resistance responses in the Russian wheat aphid-wheat interaction. J. Plant Physiol 159: 585-590

Morkunas I, Mai VC, Gabrys B (2011) Phytohormonal signaling in plant responses to aphid feeding. Acta Physiol Plant 33: 2057-2073 
Mugford ST, Barclay E, Drurey C, Findlay KC, Hogenhout SA (2016) An immuno-suppressive aphid saliva protein is delivered into the cytosol of plant mesophyll cells during feeding. Mol Plant Microbe Interact 29: 854-861

Naessens E, Dubreuil G, Giordanengo P, Baron OL, Minet-Kebdani N, Keller H, Coustau C (2015) A secreted MIF cytokine enables aphid feeding and represses plant immune responses. Curr Biol 25: 1898-1903

Nalam V, Louis J, Shah J (2018) Plant defense against aphids, the pest extraordinaire. Plant Sci 279: 96-107

Noir S, Bömer M, Takahashi N, Ishida T, Tsui T-L, Balbi V, Shanahan H, Sugimoto K, Devoto A (2013) Jasmonate controls leaf growth by repressing cell proliferation and the onset of endoreduplication while maintaining a potential stand-by mode. Plant Physiol 161: 19301951.

Poveda K, Steffan-Dewenter I, Scheu S, Tscharntke T (2003) Effects of below- and aboveground herbivores on plant growth, flower visitation and seed set. Oecologia 135:601605

Redman AM, Cipollini DF, Schultz JC (2001) Fitness costs of jasmonic acid-induced defense in tomato, Lycopersicon esculentum. Oecologia 126:380-385.

Robson F, Okamoto H, Patrick E, Harris S-R, Wasternack C, Brearley C, JG Turner (2010) Jasmonate and phytochrome A signaling in Arabidopsis wound and shade responses are integrated through JAZ1 stability. Plant Cell. 22: 1143-1160

Sattar S, Addo-Quaye C, Thompson GA (2016) miRNA-mediated auxin signalling repression during Vat-mediated aphid resistance in Cucumis melo. Plant Cell Environ 39: 12161227

Schmelz EA, Alborn HT, Banchio E, Tumlinson JH (2003) Quantitative relationships between induced jasmonic acid levels and volatile emission in Zea mays during Spodoptera exigua herbivory. Planta 216: 665-673

Shigenaga AM, Argueso CT (2016) No hormone to rule them all: interactions of plant hormones during the responses of plants to pathogens. Semin Cell Dev Biol 56: 174-189

Smith JL, De Moraes CM, Mescher MC (2009) Jasmonate- and salicylate-mediated plant defense responses to insect herbivores, pathogens and parasitic plants. Pest Manag Sci 65: 497503 
Smith CM, Liu X, Wang LJ, Liu X, Chen M-S, Starkey S, Bai J (2010) Aphid feeding activates expression of a transcriptome of oxylipin-based defense signals in wheat involved in resistance to herbivory. J Chem Ecol 36: 260-276

Staswick PE, Su W, Howell SH (1992) Methyl jasmonate inhibition of root growth and induction of a leaf protein are decreased in an Arabidopsis thaliana mutant. P Natl Acad Sci USA 89: 6837-6840

Sun M, Voorrips RE, van Kaauwen M, Visser RGF, Vosman B (2020). The ability to manipulate ROS metabolism in pepper may affect aphid virulence. Hortic Res 7: 6. https://doi.org/10.1038/s41438-019-0231-6

Takei M, Yoshida S, Kawai T, Hasegawa M, Suzuki Y (2015) Adaptive significance of gall formation for a gall-inducing aphids on Japanese elm trees. J Insect Physiol 72: 43-51

van Bel AJE, Will T (2016) Functional evaluation of proteins in watery and gel saliva of aphids. Front Plant Sci 7:1840. https://doi.org/10.3389/fpls.2016.01840

Verma V, Ravindran P, Kumar PP (2016) Plant hormone-mediated regulation of stress responses. BMC Plant Biol 16:86 DOI 10.1186/s12870-016-0771-y

Wang L, Wu J (2013) The essential role of jasmonic acid in plant-herbivore interactions - Using the wild tobacco Nicotiana attenuata as a model. J GenetGenomics 40: 597-606

Wang P, Zhao Y, Li Z, Tao WA, Xiong Y, Zhu J-K (2018) Reciprocal Regulation of the TOR Kinase and ABA Receptor Balances plant growth and stress response. Mol Cell 69, 100112

Yang D-L, Yao J, Mei C-S, Tong X-H, Zeng L-J, Li Q, Xiao L-T, SunT-P, Li J, Deng X-W, Lee CM, Thomashow MF, Yang Y, He Z, He SY (2012) Plant hormone jasmonate prioritizes defense over growth by interfering with gibberellin signaling cascade. Proc Natl Acad Sci USA 109:E1192-E1200. https://doi.org/10.1073/pnas.1201616109

Zhang Y, Turner JG (2008) Wound-induced endogenous jasmonates stunt plant growth by inhibiting mitosis. PLoS One 3: e3699. https://doi.org/10.1371/journal.pone.0003699

Züst T, Agrawal AA (2017) Trade-offs between plant growth and defense against insect herbivory: an emerging mechanistic synthesis. Annu Rev Plant Biol 68:513-34

\section{Figure legends}

Figure 1. Experimental design for plant growth determination under aphid infestation. 
Figure 2. Effect of Myzus persicae infestation on phytohormone content in pepper leaves. Means of five biological replicates \pm SD are shown. Groups not sharing a letter code were separated using Tukey ${ }^{1}$ or Games-Howell ${ }^{2}$ post hoc analysis at $P<0.05$. (A) $\mathrm{JA}^{2}$, (B) JA-Ile ${ }^{2}$, (C) $\operatorname{cisOPDA}{ }^{1}$, (D) $\mathrm{SA}^{1}$, (E) $\mathrm{ABA}^{1}$ and (F) IAA ${ }^{1}$.

Figure 3. Effect of a high density of aphid (Myzus persicae) infestation on the stem growth in pepper plants. Means of 25 plants \pm SD are shown. Asterisks indicate differences respect the control in Kruskal-Wallis test analysis at $P<0.05$.

Figure 4. Effect of aphid (Myzus persicae) infestation on the formation of new leaves in pepper plants. Means of 25 plants \pm SD are shown. Asterisks indicate differences respect the control in Kruskal-Wallis test analysis at $P<0.05$.

Figure 5. Effect of aphid (Myzus persicae) infestation on the leaf expansion in pepper plants. Means of 25 plants \pm SD are shown. Asterisks indicate differences respect the control in Kruskal-Wallis test analysis at $P<0.05$. 
電

Non-infested

Infested

4 dpi

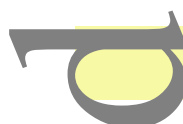

Non-infested

Infested

2 dpi

Non-infested

C
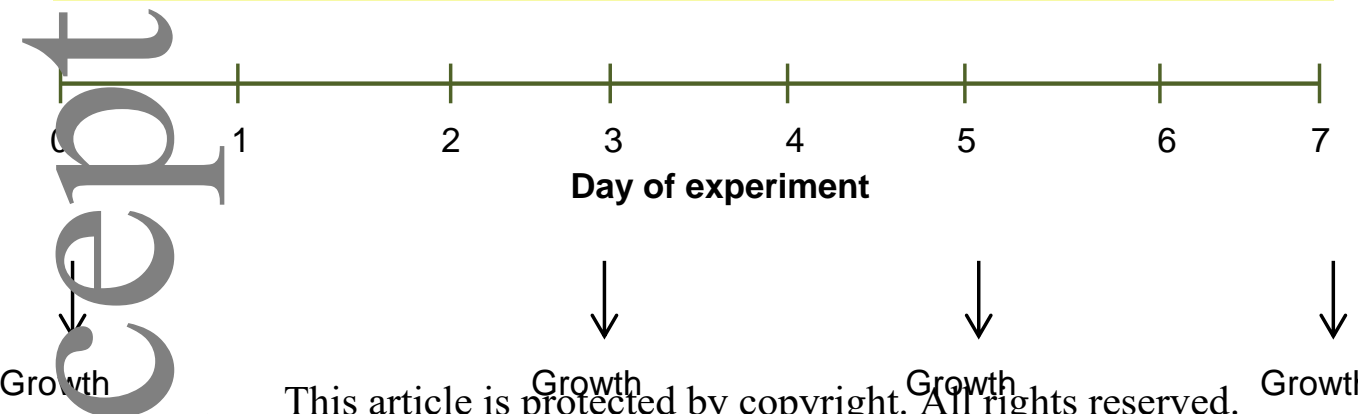

$\downarrow$

Day of experiment

Growth 

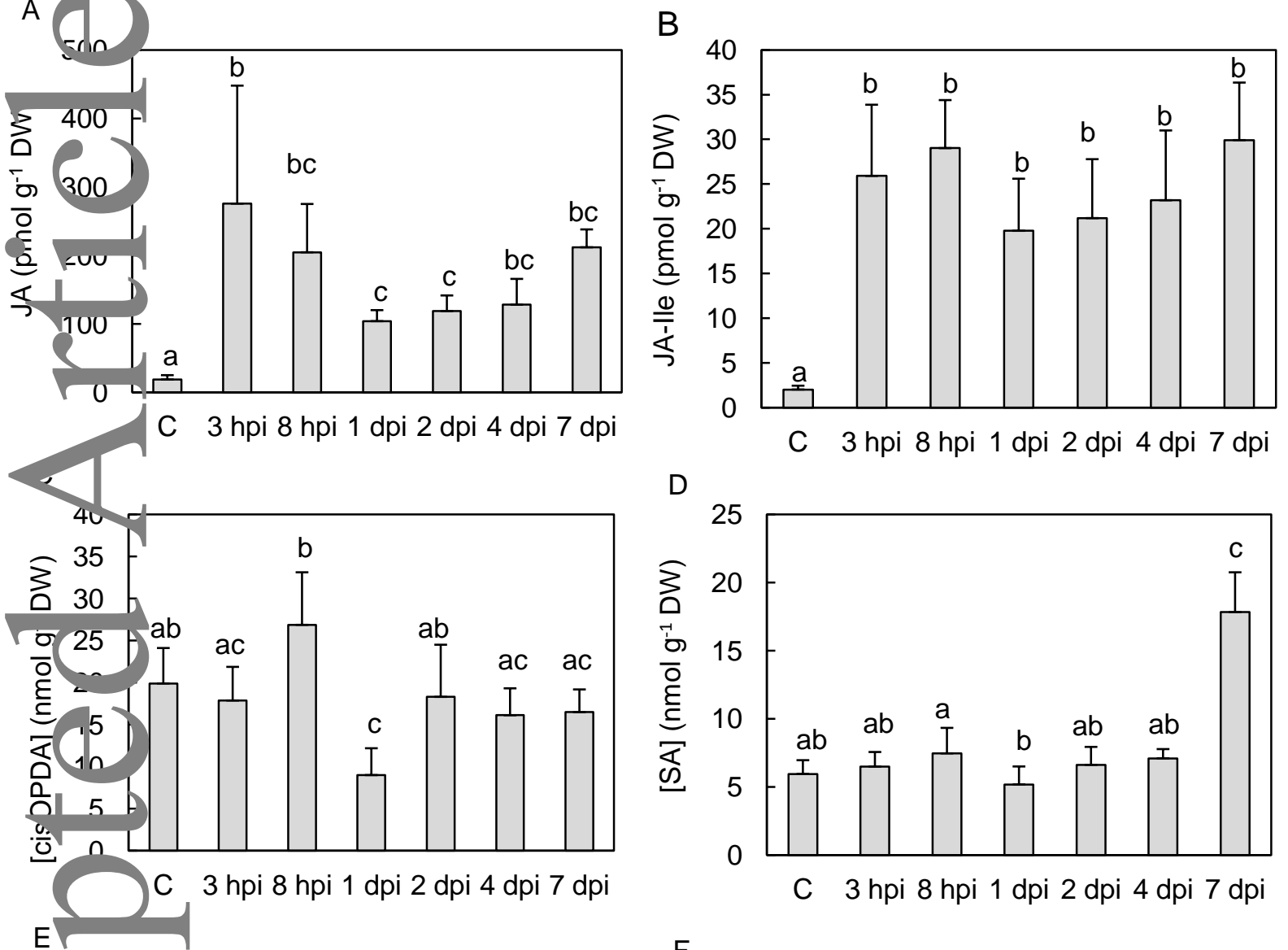

D
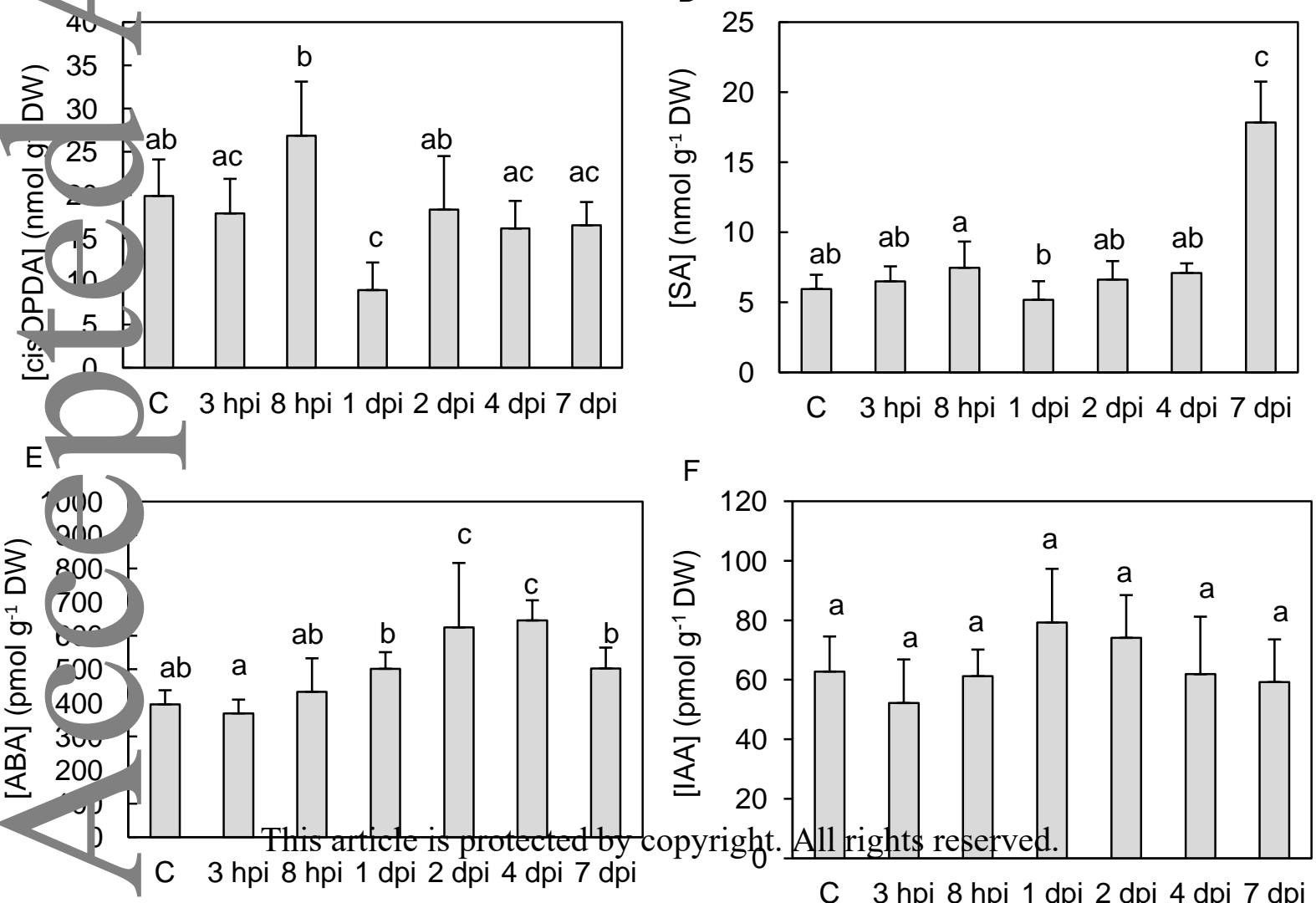

C 3 hpi 8 hpi 1 dpi 2 dpi 4 dpi 7 dpi 

